\title{
Sleep disorders in children and adolescents
}

\author{
Gregory Stores
}

The scientific study of sleep and its disorders is essentially just a few decades old, but during that time impressive advances have been made in the biological knowledge of sleep. There has been recognition and treatment of a wide range of sleep disorders from which many people in all sections of the population suffer. The common adverse consequences of persistent sleep disturbance (at personal, educational, social and occupational levels) for members of the public at large have become clear (Dement \& Mitler, 1993), as have the special risks of such problems to which certain groups are exposed - notably people with chronic psychiatric or physical disorders or learning disabilities.

However, it would never be guessed that so much is known, judging by the meagre extent to which sleep and its disorders feature in teaching and training in medicine or any of the allied health care professions. The topic is almost universally neglected - a blind spot in undergraduate, postgraduate, specialist and continuing education. For example, it is striking how little mention is made of sleep disorders and their implications in recent and otherwise well-informed textbooks of child psychiatry and paediatrics. This must mean that many opportunities to help people of all ages, who persistently suffer from sleep loss or impairment in the quality of their sleep, are missed. The adverse consequences consist not only of distress at the time of the sleep disturbance, but also detrimental effects on the various aspects of daytime functioning mentioned above. It may well be that children and adolescents are particularly vulnerable because of effects on various aspects of their development. If unresolved, early sleep problems may persist into adult life.

Support for these concerns comes from the findings of surveys of medical and paramedical professional education. The median overall teaching time devoted to sleep and its disorders in medical student teaching in the UK was recently reported to be five minutes. For each of the psychiatry and paediatric parts of the course the figure was also five minutes and zero minutes for some other parts of the course including general practice (Stores \& Crawford, 1998). The situation is no better for both undergraduate and clinical psychology teaching and training in the UK (Wiggs \& Stores, 1996; Stores \& Wiggs, 1998), which is unfortunate in view of the important part that psychologists can play in the treatment of sleep disorders. It is cold comfort that, despite the generally greater awareness of sleep disorders in the USA, a similar situation has been reported there in medical student education (Rosen et al, 1993), various medical specialities (Moline \& Zendell, 1993) and both undergraduate and graduate nurse training (Cohen et al, 1992). Mainland Europe has the same problems (Salzarulo, 1990).

There is, therefore, an apparently universal need to correct these educational deficiencies. In the UK, the Royal Medical Colleges can reasonably be expected to take a lead regarding the content of their recommendations in teaching and training, and also their examinations in which sleep and sleep problems are represented very sparsely. For psychiatrists and other groups, sleep and its disorders lends itself very well as a topic for coordinated teaching as recommended for medical student courses by the Education Committee of the General Medical Council (1993). Important general themes can be emphasised, such as: the wideranging (even economic) aspects of the illness or disorder; the need to integrate basic medical science and clinical issues; the inter-speciality and interdisciplinary nature of accurate diagnosis and

Gregory Stores is Clinical Reader in Child Neuropsychiatry at the University of Oxford Department of Psychiatry, Park Hospital for Children, Old Road, Headington, Oxford OX3 7LQ. His background includes psychology, general medicine, neurology, clinical neurophysiology and psychiatry. His previous clinical activities were principally in childhood epilepsy and EEG. In recent years his research interests have been in sleep disorders. 
effective management; and developmental aspects of the nature, presentation, management and prognosis of the illness or disorder from infancy to old age. There is no problem defining an appropriate and realistic syllabus for the various professional groups. Apart from certain basic considerations, the nature and the emphasis would need to be different in each case but with the stress on comprehensive care whatever the age of the patient.

\section{General aspects}

\section{Conventional accounts are misleading}

As part of the general neglect of sleep disorders, many child psychiatry and paediatrics textbook accounts do not convey the frequency and range of disorders in young people. The range of treatment options is also generally underestimated.

Studies of children and adolescents in the general population have indicated that $20-30 \%$ of this group have sleep problems of concern to parents or themselves. Such problems take many forms which can be grouped into three general categories: sleeplessness, excessive sleepiness and night-time episodes of disturbed behaviour (parasomnias). A distinction needs to be made between a sleep problem (or complaint) and the underlying sleep disorder - there can be many possibilities for a given sleep problem. Over 80 sleep disorders are described in the International Classification of Sleep Disorders (American Sleep Disorders Association, 1997) many of which are encountered in children and adolescents. With an accurate diagnosis, a rational choice can be made from the many forms of treatment that have been developed, for instance, behavioural, cognitive, chronobiological, pharmacological and even surgical treatments in certain conditions. The application of these approaches will be mentioned later in relation to specific sleep disorders. At present they are generally under-utilised because their need or availability is not realised.

\section{Crossing traditional boundaries of diagnosis and treatment}

Sleep is affected by many factors such as development, intrinsic physiological systems and processes, the physical and psychological environments and also mental state. Sleep disorders can be caused, precipitated or maintained by unsatisfactory parenting, interpersonal or family problems, physical illness or psychiatric disorder. In the case of the sleep disorders of childhood and adolescence, it is necessary to draw on aspects of general paediatrics, child neurology and respiratory medicine, developmental psychology and also child and adolescent psychiatry including its family aspects. It is one of the fascinations of the sleep disorders field (but also a reason for its relatively late progress and limited impact so far in teaching and research) that it requires this multi-speciality and multi-disciplinary integration.

\section{Differences between child and adult sleep disorders}

A special approach is required for the recognition, definition and treatment of sleep disorders in young people.

Parents usually decide that their child's sleep is a problem. Their concerns are influenced by their expectations (sometimes misinformed), preferences, marital state and other factors. All of these need to be assessed in deciding the nature of the problem and what needs to be done to help. Conversely, parents may not realise that their child's sleep is disturbed, or they may sometimes be indifferent to the idea of treatment because they mistakenly see the problem as inevitable.

Parenting and other aspects of family life, for example, over-indulgence or inconsistency, feature prominently in the origins of many sleep disorders in early childhood, even those caused by physical illness or disorder. Such factors also affect the implementation of treatment programmes. However, many forms of childhood sleep disorders can be treated successfully, possibly more so than those in adult life which have become well established over many years.

The pattern of sleep disorders is different in children and adolescents compared with adults. Sometimes this reflects developmental changes in sleep physiology, for example, parasomnias arising in deep non-rapid eye movement (non-REM) sleep are particularly common in young children because of the greater depth and duration of that form of sleep at that stage of development.

The significance of many parasomnias can vary considerably with age. Sleep terrors in childhood are usually a transient phenomenon of no pathological significance; in adult life it is the conventional view that they may indicate underlying psychological disturbance; onset in old age may suggest an organic pathology.

The cause, manifestations, diagnostic criteria and treatment requirements of basically the same sleep disorder can be very different in children compared with adults. Obstructive sleep apnoea is a striking 
example. In contrast to the typical adult cases, children with obstructive sleep apnoea are not usually obese and, indeed, may have failed to thrive; tonsils and adenoids are the usual cause, necessitating removal; the gender ratio is equal; and daytime overactivity and other disruptive behaviour may result, rather than excessive sleepiness. Physiological diagnostic criteria derived from adult studies do not always apply to children. Important differences between children and adults are also seen in the clinical presentation and polysomnographical features of narcolepsy.

\section{Links between sleep disorders and child psychiatry}

The neglect of sleep disorders in teaching and training in psychiatry is particularly unfortunate because the need to consider such disorders in clinical practice is important. This is true whatever the patient age group, but especially so in child and adolescent psychiatry (see Box 1).

Sleep disturbance is particularly prominent in children and adolescents with psychiatric problems (Salzarulo \& Chevalier, 1983; Simonds \& Parraga, 1984). This is true not only of severe depression (Ryan et al, 1987), but also in association with many other conditions (Stores, 1996) such as conduct disorders and oppositional behaviours, anxiety states, attention deficit hyperactivity disorder, chronic fatigue syndrome, drug misuse, following trauma, and autism (Stores \& Wiggs, 1998). Children with a learning disability show particularly high rates of sleep problems (Stores, 1992). The same applies to children with chronic physical illness (a fact of special importance for psychiatrists involved in paediatric liaison work). This is partly because of the emotional consequences of being ill and perhaps

Box 1. Importance of sleep disorders in child and adolescent psychiatry

Sleep disturbance commonly complicates psychiatric disorder

Sometimes sleep disturbance is a cause of or contributory factor to psychiatric disturbance

Sleep disorders can be misconstrued as primary psychiatric disorder and vice versa

Children's sleep disturbance may cause or be caused by their parents' psychiatric problems hospitalised (White et al, 1990), but also because certain illnesses, or possibly their treatment, directly affect sleep. Asthma is a good example (Stores et al, 1998).

The precise nature of the sleep disturbances in child and adolescent psychiatric disorders largely remains to be determined. They tend to be reported in terms of sleep problems rather than specific disorders. At the physiological level there are conflicting reports about sleep stage (especially REM sleep) abnormalities in severe depression comparable to the biological markers reported in adults.

Although in many of the above examples sleep disruption can be seen as a consequence, in some instances it might be the cause of, or at least a factor which contributes to or exacerbates, the psychological disturbance.

There is good experimental and clinical evidence that many aspects of mood, behaviour and cognitive function are affected in adults by sleep loss, impaired quality of sleep, or inappropriate timing of the sleep period (Bonnet, 1994; Pilcher \& Huffcut, 1996). The same seems likely to be true of children (further details available from the author upon request).

The range of adverse cognitive effects is wide, and includes impaired attention (especially sustained attention) and memory. Creative ability is also thought to be particularly vulnerable. Consequently, school work is likely to suffer and proneness to accidents may be increased. Mood changes have also been reported in the literature as sometimes causing irritability and aggression. It has been argued that, for various reasons connected with a modern Westernised lifestyle, a large proportion of the general population is chronically deprived of sleep. Adolescents appear to be at special risk of sleep deprivation and its psychosocial consequences and risks because, although their sleep requirements increase for physiological reasons, they actually get less sleep for social and other reasons (Carskadon, 1990).

It is easy to see how coping with difficulties, including pre-existing psychological problems, will be affected by persistent sleep disturbance. As yet, it remains largely an open question (which needs to be explored) how far psychiatric disorder can be primarily attributed to a sleep disorder. There are suggestions that this may be so in some cases of attention deficit hyperactivity disorder (Stores, 1992). Of course, children are likely to be emotionally upset (or even frightened) by aspects of the sleep disorder, depending on its type and the effects on others, as well as the restrictions it imposes. For example, depressive symptoms and behavioural disturbance are common consequences of narcolepsy or delayed sleep phase syndrome.

A very practical reason why psychiatrists should be familiar with the sleep disorders field is that, if they are not, diagnostic errors may readily occur. 
Sleep disorders can be mistaken for a primarily psychological problem. This happens repeatedly in childhood narcolepsy where long delays in the correct diagnosis are common, the disorder often being misconstrued as laziness (Guilleminault \& Pelayo, 1998); otherwise, a serious psychiatric disorder may be misdiagnosed (Dahl et al, 1994). Other examples of such mistakes include the KleineLevin syndrome (Pike \& Stores, 1994). More commonly the psychological difficulties of getting to sleep and waking at the required time, characteristic of delayed sleep phase syndrome, are misconstrued as 'difficult' behaviour by parents and doctors alike. Such delays and confusions in diagnosis (with inappropriate and therefore disappointing advice or treatment) are highly likely to increase the stress experienced by both the child and the family.

This last point highlights the connections that can exist between children's sleep disorders and their parents' psychological problems.

The relationship can be in either direction. Longstanding sleep disturbance can have a damaging effect on parenting ability and other aspects of family life. Quine (1992) found that mothers of children with a learning disability and severe sleep problems were more irritable, concerned about their own health and less affectionate towards the children, with less control and increased use of physical punishment, than mothers of such children without sleep problems. Initial research suggests that mother-child relationships, maternal mental state and confidence in parenting ability, as well as the child's behaviour, improve if the child can be helped to sleep better (Wolfson et al, 1992; Minde et al, 1994).

Although there has been interest in the effect of parental (mainly maternal) psychiatric illness (notably depression) on various aspects of child development, its possible effects on sleep patterns appear to have been largely overlooked. Only very preliminary empirical findings have been published which do not allow any firm conclusions to be drawn (Seifer $e t$ al, 1996). At a less serious level of maternal disturbance, recent research has provided some support for the notion that emotional and attachment issues influencing maternal cognition about the child are associated with sleep disturbance in infants (Morrell, 1998).

\section{Basic aspects of assessment}

A significant reason why sleep problems and their causes are overlooked in psychiatry and other specialities is that conventional history-taking contains very little about sleep and its disorders.
Basic screening questions should be asked about any child concerning the following:

(a) difficulty getting to sleep or staying asleep;

(b) excessive sleepiness during the day;

(c) disturbing episodes at night.

Positive answers call for a detailed sleep history which is the cornerstone of sleep assessment. The main aspects are shown in Box 2 .

A suggested schedule for reviewing a child's 24hour sleep-wake cycle is provided in the Appendix. The items need to be modified somewhat according to the age of the child.

Additional aspects of the overall review of a child with a sleep problem in order to identify possible causes are: developmental history (including delays, illnesses, and traumatic experiences); general health; physical examination; behaviour and emotional state; and family history and circumstances. A sleep diary kept for two weeks or more often provides a more complete and balanced account of the child's sleep patterns and problems than the clinic interview.

Only a minority of children need polysomnography (physiological sleep studies). This entails recording electroencephalogram (EEG), eye movements and muscle activity from which the different stages of sleep can be identified. Home polysomnography systems are particularly appropriate for children (Stores, 1994). The main uses of polysomnography are the investigation of excessive daytime sleepiness and complicated parasomnias. Recording of body movements alone (actigraphy) can provide useful information on basic sleepwake patterns. Other special investigations may be required, depending on the nature of the problem.

\footnotetext{
Box 2. Main aspects to be covered in a sleep history

Current sleep problem and its evolution Past treatments and their effects

Review of the child's current 24-hour sleepwake cycle in order to determine in particular: duration of sleep; quality of sleep (continuous or disrupted); timing of sleep and features suggestive of specific sleep disorders

Sleep environment and arrangements

Development of the child's sleep patterns and problems

Family history of sleep problems
} 


\section{Nature and treatment of sleep disorders}

The following is a selective account of the many sleep disorders in young people, with an emphasis on aspects which are particularly relevant to clinical practice in child and adolescent psychiatry. It is hoped that the three articles on sleep disorders in this issue of APT will all be read in relation to each other to help promote a whole-life, developmental approach to sleep disorders rather than a preoccupation with a given agegroup. The disorders are described in relation to the three main categories of sleep problem mentioned earlier. Often children have more than one current sleep problem; also the nature of the problems may change with age. Further information on sleep physiology and the precise details of investigation and treatment and other aspects can be found in the books edited by Ferber \& Kryger (1995) and Dahl (1996), and the reviews by Stores (1996) and Rosen (1997).

\section{Sleeplessness}

This very general term covers such problems as reluctance to go to bed, difficulty getting off to sleep, recurrent night wakenings or otherwise disturbed sleep, early morning waking and short duration of sleep. The nature of the sleeplessness must be determined precisely as different factors may be responsible and different treatment needed in each type of sleeplessness, although combinations of these problems are not uncommon. Close enquiry sometimes reveals that the child's sleep pattern is unremarkable for its age, in which case parents need this to be explained to them. It may be useful to refer to the normative data that have been compiled for typical sleep duration at different ages (see Table 1) realising, however, that individual differences are prominent from an early age. More substantial help will be required if the parents' view of the situation is distorted by their own psychological problems.

Most parents prefer the child to sleep separately from themselves. Therefore, it is appropriate from an early age (6-9 months) to encourage the child to settle to sleep, and also to return to sleep when he or she wakes at night (a normal phenomenon), without his or her parents' presence and attention. A child's ability to do this is referred to as 'self-soothing'. This is not achieved in about $20 \%$ of 1- to 3-year-olds, often causing much distress (or worse) where parents have other serious problems to contend with. For example, the possibility of non-accidental injury in these circumstances has been raised by Chavin \& Tinson (1980).
Table 1. Nerage sleep duration at different ages

$\begin{array}{lc}\text { Age } & \text { Sleep durat } \\ \text { Full-term newborn baby } & 16 \text { hours } \\ 2 \text { years } & 13 \text { hours } \\ 5 \text { years } & 11 \text { hours } \\ 9 \text { years } & 10 \text { hours } \\ 14 \text { years } & 9 \text { hours } \\ 17 \text { years } & 8 \text { hours }\end{array}$

A wide range of factors may be relevant to the onset or persistence of sleeplessness (see Box 3).

Possible medical causes of discomfort or distress must be considered and relieved whenever appropriate, but usually parenting practices or emotional upset underlie the problem. This may begin with overfeeding the infant at night causing discomfort from wet nappies, and also delay in establishing the circadian pattern of sleeping at night and feeding during the day. At a later stage an inappropriate pattern of napping (too little, too much or too near bedtime) can cause sleeping difficulties at night.

Although commonly prescribed, medication has little part to play - behavioural treatments are often very successful, even in very severe and longstanding problems of this type including those in children with a learning disability (Wiggs \& Stores, 1998). Overall, active intervention for these behavioural' sleep disorders is required, preferably at an early stage rather than waiting for spontaneous improvement as much harm can be caused to the child and the family in the meantime. Basic aims include a consistent bedtime routine (easier specified than achieved in the more chaotic families or with overindulgent parents), and the child's ability to go to sleep alone. This second aim can be

Box 3. Factors to consider in childhood sleeplessness (depending somewhat on age of child)

Medical conditions

Parenting practices (includes failure to set limits)

Disturbing or inappropriate sleep onset associations (including night-time fears and parental attention)

Delayed sleep phase syndrome (especially adolescents)

Psychological disorder, medication or illicit drug effects

Generally poor sleep hygiene 
provided by a sleeping environment with familiar and pleasant 'sleep onset associations' which are the same when the child goes to sleep and when he or she wakes in the night.

Where necessary, parents must be helped to set limits to their child's delaying tactics about going to bed, and to avoid inadvertently reinforcing the problem behaviour by acceding to repeated requests for food or comfort. The bedroom should not become associated with arousing activities, emotional upset or with punishment. Star charts and positive rewards can be useful measures for children over the age of three. The specific ways in which these behavioural approaches can be applied are described by France $e t$ al (1996) and in a very practical way for parents by Quine (1992). In general, methods involving gradual change are preferable but success can often be achieved quickly.

Difficulty getting to sleep or returning to sleep are commonly caused by night-time fears of the dark, monsters, or in older children, anxiety about daytime experiences, their parents' safety or even dying in their sleep. Comfort and reassurance are usually sufficient until the child grows out of this phase. However, more definitive psychiatric attention is required if the fears prove to be part of more serious disorder. For example, following tragic events or, in some cases, abuse, where bedtime has become associated with distressing experiences.

Sleep onset difficulties are a salient feature of delayed sleep phase syndrome which is particularly common in adolescents although it is encountered throughout childhood and adult life. For social and other reasons (including persistent sleep onset difficulties of behavioural origin, illness or holidays) the child develops the habit of not going to sleep until very late and eventually, because of a shift in the sleep phase, this becomes a physiological inability to fall asleep at the desired earlier time. In an attempt to meet sleep requirements, there is a need to sleep until late in the morning (or even later) if possible. Getting up for school becomes particularly difficult. In fact, great difficulty getting up in the morning is the main complaint in delayed sleep phase syndrome.

Preferably with the help of sleep diary information over 3-4 weeks, the characteristic delayed sleep phase syndrome features to recognise are: persistently severe difficulty getting to sleep, sound sleep, short duration of sleep on school days, but sleepingin until very late at weekends, and normal sleep in the holidays, but with a the delay in the sleep phase. Especially in the first part of the day, sleepiness and under-functioning is prominent, giving way to alertness in the evening and the early hours of the morning.

Both lateness in going to sleep and difficulty in getting up in the morning are easily misinterpreted as difficult behaviour; resetting the biological clock is required rather than recrimination. With cooperation this can be achieved by regular sleep schedules every day and good sleep hygiene. In resistant cases of delayed sleep phase syndrome 'chronotherapy' is required, that is, gradually advancing sleep onset time or (if the delay is more serious) progressively delaying it by a further three hours each 24-hour period (Sheldon et al, 1992). Other possible measures include bright light exposure or melatonin, although the effectiveness and safety of this drug is uncertain. Sometimes the sleep phase delay pattern is preferred by the young person as a means of avoiding stressful circumstances at school or within the family. In these complicated circumstances (which might well give rise to social isolation, poor school performance, depression or substance misuse) more vigorous psychiatric help will be required.

Other causes of difficulty in going to sleep, broken or short duration sleep, especially in adolescence, include: anxiety, depression, recreational drugs (caffeine, nicotine, alcohol) and the use of illicit substances (including withdrawal effects), each requiring its own specific intervention.

Special difficulty in getting to sleep can become conditioned and persistent after the original reason no longer exists.

'Sleep hygiene' should be encouraged in patients with any form of sleep disturbance. This can be therapeutic in itself and it is an important adjunct to more specific treatments. Basic principles for children and adolescents are suggested in Box 4. Their appropriateness varies with age. Several of them are particularly relevant to older children or adolescents who complain about lying in bed awake for long periods unable to sleep, although the specific causes just mentioned should be considered. It is important that bed does not come to be associated with difficulty sleeping. If the individual does not fall asleep after about 20 minutes it is better for them to move to another room for a while until they become tired enough to sleep.

\section{Excessive daytime sleepiness}

Excessive daytime sleepiness is possibly the most overlooked or misconstrued category of sleep disturbance, especially in young people, because it is very easily mistaken for laziness, disinterest, lack of motivation, depression or even intellectual limitation.

Such errors can only be avoided by considering the possibility of a sleep disorder in any young person who appears to be under-functioning, and also by being familiar with the characteristic features of the possible sleep-related causes discussed below. 
Box 4. Sleep hygiene principles for children and adolescents (relevance varies according to age)

\section{Sleeping environment}

Familiar, comforting setting

Comfortable bed

Correct temperature

Darkened, quiet room

Non-stimulating

Non-punitive

\section{Encourage}

Bedtime routines

Consistent bedtime and waking-up times

Falling asleep without parents

Going to bed only when tired

Sort out problems and plans before bedtime

Avoid

Boisterous play or other over-excitement near bedtime

Caffeine-containing drinks late in the day

Even when excessive sleepiness is perceived as such, it may not be viewed as a medical problem for which treatment is available (based on the underlying sleep disorder).

An abnormal tendency to sleep should be distinguished from fatigue, exhaustion or lack of energy for which different explanations are likely, such as anaemia or other physical illness. Such physical factors, however, should be excluded in cases of genuine excessive sleepiness.

Whereas sleepiness usually implies a lowering of activity levels, in young children it may produce irritability and overactive behaviour not readily recognised as the result of sleep disturbance (Stores, 1992).

Excessive daytime sleepiness is considered to be a serious problem for about $5 \%$ of adults. The number of affected children and adolescents is not known, but it is a feature of many conditions or circumstances encountered at an early age. The basic causes can be grouped as listed in Box 5 .

\section{Insufficient sleep}

Sleeplessness is the most common cause of excessive daytime sleepiness at all ages, but adolescents are at special risk because of their increased need for sleep at a time when social activities or study reduce the likelihood that they will sleep sufficiently long or regularly enough. Many teenagers actually complain of being tired, but many more than this are likely to be suffering from sleep deprivation ('chronic sleep debt') without it being realised. The use of stimulants to stay awake, or alcohol to help sleep onset, complicates the situation further.

Because it appears to be so common, delayed sleep phase syndrome should readily be considered in young people unable to get to sleep, combined with great difficulty getting up in the morning and possibly falling asleep during the day.

At any age, sleep can be insufficient because of irregular or erratic sleep-wake schedules. This may be a feature of the family's generally poor routine but, again, lifestyle may well be the cause, especially in adolescence.

\section{Disturbed overnight sleep}

The duration of sleep and timing of the sleep phase may be satisfactory but the restorative value (or quality) of sleep may be impaired by frequent disruption.

The many possible causes include medical conditions in which symptoms can intensify in sleep (e.g. asthma) or where pain or discomfort interrupt sleep. Some degree of upper airway obstruction during sleep (including actual obstructive sleep apnoea) is said to occur in at least $1 \%$ of children in the general population. It is particularly common in children with neurological disorders (notably Down's syndrome, the mucopolysaccharidoses, some cases of the Prader-Willi syndrome and fragile $X$ syndrome, neuromuscular disorders, cerebral palsy and brain stem abnormality associated with spina bifida and hydrocephalus), as well as those with craniofacial syndromes or other diseases which impinge on or infiltrate the upper airway, including sickle cell disease. Important signs include snoring (although only about one in 5-10 children who snore have significant upper airway obstructions), other

Box 5. Possible causes of excessive daytime sleepiness

Insufficient sleep caused by sleeplessness, irregular sleep-wake cycle, delayed sleep phase syndrome

Disturbance of overnight sleep, e.g. by medical conditions (including obstructive sleep apnoea), frequent parasomnias, drug or alcohol effects

Increased sleep requirements, e.g. narcolepsy, Kleine-Levin syndrome 
evidence of breathing difficulty during sleep (including paradoxical movements of the chest and abdomen during respiration), very restless sleep, neck extension during sleep, diffuse sweating during sleep, evidence of mouth breathing and possibly nocturnal enuresis.

As the repeated disturbance of sleep physiology in upper airway obstruction can cause sleepiness and significantly affect daytime learning and behaviour, relief of the obstruction is important wherever possible. Children in the general population may well benefit from removal of enlarged tonsils and adenoids (the usual cause of obstruction); in other groups where the cause of the obstruction is more complicated, treatment possibilities are fewer but should be attempted based on careful assessment of the individual case.

Various other psychological and pharmacological causes of broken sleep have been mentioned. Frequent parasomnias also disturb sleep. Disruption of sleep by periodic limb movements is thought by some to be more common in children than previously supposed.

\section{Conditions in which sleep requirements are increased}

Here the excessive sleepiness is an intrinsic part of the condition rather than a consequence of something else.

The main example of persistent excessive sleepiness of this type is narcolepsy, which is by no means rare (4-7 per 10000$)$ and very often starts by adolescence. Early diagnosis is unusual for a number of reasons. Initial signs can be non-specific (including prolongation of overnight sleep without sleep attacks); the features of cataplexy may be subtle and delayed; other features may be denied; adultbased polysomnographical criteria do not necessarily apply; and the overall picture may be obscured by the child's emotional reaction to the condition.

It is appropriate to consider narcolepsy in any young person who is excessively sleepy during the day without an obvious explanation such as short duration of sleep or a clearly disrupted sleep-wake pattern. Repeated assessment is often needed before a definite diagnosis can be made on the basis of emerging clinical and polysomnographical features characteristic of the condition. Narcolepsy is a very persistent and disabling condition for which careful treatment with medication and ancillary measures, as well as much support, is needed.

In Kleine-Levin syndrome, which also usually begins in the teenage years, excessive daytime sleepiness is intermittent with normality in between, and associated (in its classical form) with overeating, hypersexuality and other forms of disturbed behaviour. The nature of this condition is mysterious, but it has various clinical and polysomnographical affinities with major affective disorders. Other possible causes of intermittent excessive daytime sleepiness include depression (in which the sleepiness is modest compared with the KleineLevin syndrome), drug-induced states and menstruation-related sleepiness.

\section{Parasomnias}

Episodic disturbances of behaviour or experiences associated with sleep are especially common in childhood. The more dramatic varieties are a particular source of concern to parents and physicians alike who often consider that they indicate an underlying psychological problem. This is rarely the case, in contrast (it is thought, although not well demonstrated) to their occurrence into adult life.

In most cases, parents can be reassured that their child will grow out of the condition and that psychiatric intervention is not warranted, although some practical measures may be required, for example, avoiding accidental injury during sleepwalking. However, it is important to try to identify the exceptions to this general rule that childhood parasomnias are benign. Research on this point is very limited, but frequent occurrence, late onset or recurrence or onset following trauma (sometimes covert) are possible pointers to the need for further enquiries.

A major problem with the parasomnias is the tendency to confuse them with each other; the more dramatic forms may all be referred to as 'nightmares'. In fact, there are distinct types of parasomnias grouped according to their relationship to different sleep stages or, in some cases, the physical or psychiatric disorder of which they are a manifestation. The following account is confined to some of the parasomnias of particular psychiatric importance in childhood and adolescence (see Box 6).

Sleep-walking, night terrors (and the less wellknown confusional arousals) are all so-called partial arousal disorders because they occur when there is a sudden change from deep non-REM sleep to a later stage of sleep. As deep non-REM sleep is most abundant in the first third of the night, these conditions tend to occur in that period. They involve a curious combination of appearing to be awake but actually still being asleep. The apparent degree of distress is greatest in night terrors, but sleepwalking may also take an agitated form. Confusional arousals (usually in younger children) might also be distressing for parents who understandably (although mistakenly) think that the child is suffering during the episode. The child's behaviour 
may be complicated, apparently purposeful and occasionally violent. Older children may wake up at the end of an episode and, in the case of night terrors, describe a brief and fragmentary feeling of danger or threat.

However dramatic the events, these arousal disorders do not usually signify any psychological disturbance. Affected children often seem to share with other members of their family a genetic predisposition which resolves spontaneously with time. Explanation and reassurance is, therefore, usually appropriate although practical measures may also be necessary such as avoidance of accidental injury. Other helpful measures include avoidance of overtiredness, promoting a relaxed attitude to bedtime, and help with any daytime worries. Medication is reserved for particularly severe cases.

Nightmares are also very common, although they occur only occasionally. As they arise in REM sleep they occur later in the night when this type of sleep is most abundant. In contrast to partial arousals, the child wakes, is able to recall the narrative of his or her frightening dream, and can be comforted. Nightmares and bedtime fears may coexist. Their content may indicate the source of stress which has precipitated them. Treatment involves avoiding obvious causes, or more formal psychiatric help if the nightmares are part of a serious condition such as post-traumatic stress disorder.

Sleep-related head-banging or associated rhythmic movements occur in many young children and are usually interpreted as a self-comforting way of getting to sleep or returning to sleep. However, parents may well be very concerned about this strange behaviour and worried about possible injury if the child seeks out a hard surface against which to bang his or her head. In fact (unlike daytime headbanging which is associated with severe developmental delay or psychiatric disorder), such behaviours at night are also benign and usually resolve

Box 6. Common or important causes of recurrent disturbance of behaviour or experience associated with sleep

Partial arousal disorders (sleep-walking, night terrors, confusional arousals)

Nightmares

Head-banging and other rhythmic movements

Hypnagogic or hypnopompic imagery

Nocturnal epileptic seizures spontaneously by 3-4 years of age. Protective measures may be appropriate in the meantime, or behavioural treatment in particularly troublesome cases.

Other parasomnias include the common problems of nocturnal enuresis (of which there are good accounts in standard textbooks, e.g. Graham (1991)); sleep-walking, which is common and usually not significant; and hypnagogic and hypnopompic imagery, which are common and also usually unassociated with other problems, although they can be frightening to children. It is important to recognise nocturnal epilepsy and not to confuse it with other parasomnias because of its very different significance (Stores, 1991). The diagnosis of nocturnal epilepsy is made more likely by the occurrence of similar attacks during the day. However, especially in certain types of epileptic seizure, the distinction can be difficult and careful physiological monitoring may be required.

\section{Conclusions}

All psychiatrists need to be familiar with the diagnostic and treatment principles of sleep disorders medicine because sleep disturbance features so prominently in their work in many ways. This is at least as true for child and adolescent psychiatry as for other branches of the speciality. The sleep disorders field is advancing rapidly and offers many opportunities for accurate diagnosis and successful treatment to the benefit of members of the general public, as well as those whose psychiatric or physical problems are complicated by sleep disturbance and its consequences.

\section{Appendix}

Review of child's 24-hour sleep-wake cycle (modified according to child's age).

\section{Evening}

- Time of evening meal

- Other evening activities

- Going to bed

- Preparation for bed, by whom

- Time of going to bed 
- Reluctance to go at required time, parents' reactions

- Fears, rituals

- Wanting to sleep with someone, other comforts

- Time taken to fall asleep, other experiences during the period

\section{When asleep}

- Wakings, frequency, causes, ability to return to sleep

- Episodic events, exact nature, timing, frequency

- Other behaviours during sleep, for example, snoring, restlessness, bed-wetting

- Parents' reaction to night-time events

\section{Waking}

- Wakes spontaneously or needs to be woken up

- Time of final waking

- Total duration of sleep period

- Longest period of uninterrupted sleep

- On waking: preoccupations, mood, feeling of being refreshed, other experiences

- Difficulty getting out of bed, time of getting out of bed

\section{Daytime}

- Sleepiness, naps

- Lethargy

- Mood

- Overactivity

- Concentration and performance

- Other unusual episodes

\section{References}

American Sleep Disorders Association (1997) International Classification of Sleep Disorders, Revised: Diagnostic and Coding Manual. Rochester, MN: ASDA.

Bonnet, M. H. (1994) Sleep deprivation. In Principles and Practice of Sleep Medicine (2nd edn) (eds M. H. Kryger, T. Roth \& W. C. Dement), pp. 50-67. Philadelphia, PA: W. B. Saunders.
Carskadon, M. A. (1990) Adolescent sleepiness: increased risk in a high risk population. Alcohol, Drugs and Driving, 5, 317-327.

Chavin, W. \& Tinson, S. (1980) Children with sleep difficulties. Health Visitor, 53, 477-480.

Cohen, F. L., Merritt, S. L., Nehring, W. M., et al (1992) Curricular sleep content in graduate and undergraduate nursing programs. Sleep Research, 21, 187.

Dahl, R. (ed.) (1996) Sleep disorders. Child and Adolescent Clinics of North America, 5, 543-767.

—, Holttum, J. \& Trubnick, L. (1994) A clinical picture of child and adolescent narcolepsy. Journal of the American Academy of Child and Adolescent Psychiatry, 33, 834-841.

Dement, W. C. \& Mitler, M. M. (1993) It's time to wake up to the importance of sleep disorders. Journal of the American Medical Association, 269, 1548-1550.

Education Committee of the General Medical Council (1993). Tomorrow's Doctors. Recommendations on Undergraduate Medical Education. London: GMC.

Ferber, R. \& Kryger, M. H. (eds) (1995) Principles and Practice of Sleep Medicine in the Child. Philadelphia, PA: W. B. Saunders.

France, K. G., Henderson, J. M. T. \& Hudson, S. M. (1996) Fact, act and tact: a three-stage approach to treating the sleep problems of infants and young children. Child and Adolescent Psychiatric Clinics of North America, 5, 581-600.

Graham, P. (1991) Child Psychiatry: A Developmental Approach (2nd edn). Oxford: Oxford University Press.

Guilleminault, C. \& Pelayo, R. (1998) Narcolepsy in prepubertal children. Annals of Neurology, 43, 135-142.

Minde, K., Faucon, A. \& Falkner, S. (1994) Sleep problems in toddlers, effects of treatment on their daytime behavior. Journal of the American Academy of Child and Adolescent Psychiatry, 33, 1114-1121.

Moline, M. L. \& Zendell, S. M. (1993) Sleep education in professional training programs. Sleep Research, 22, 1.

Morrell, J. M. B. (1998) The role of maternal cognitions in infant sleep disorder as assessed by a new instrument, the Maternal Cognitions Questionnaire. Journal of Child Psychology and Psychiatry, in press.

Pike, M. \& Stores, G. (1994) Kleine-Levin syndrome: a cause of diagnostic confusion. Archives of Disease in Childhood, 71, 355-357.

Pilcher, J. J. \& Huffcutt, A. I. (1996) Effects of sleep deprivation on performance: a meta-analysis. Sleep, 19, 318-326.

Quine, L. (1992) Severity of sleep problems in children with severe learning difficulties: description and correlates. Journal of Community and Applied Social Psychology, 2, 247268.

Rosen, C. I. (1997) Sleep disorders in infancy, childhood and adolescence. Current Opinion in Pulmonary Medicine, 3, 449455.

Rosen, R. C., Rosekind, M., Rosevaar, C., et al (1993) Physician education in sleep and sleep disorders: a national survey of US medical schools. Sleep, 16, 249-254.

Ryan, N. D., Puig-Antich, J., Rabinovich, H., et al (1987) The clinical picture of major depression in children and adolescents. Archives of General Psychiatry, 44, 854-861.

Salzarulo, P. (1990) Workshop on education about sleep in Europe: chairman's summary. In Sleep '90. Proceedings of the Tenth European Congress on Sleep Research (ed. J. A. Horne), pp. 475-478. Bochum: Pontnagel Press.

- \& Chevalier, A. (1983) Sleep problems in children and their relationships with early disturbances of the wakingsleeping rhythms. Sleep, 6, 47-51.

Seifer, R., Sameroff, A. J., Dickstein, S., et al (1996) Parental psychopathology and sleep variation in children. Child and Adolescent Psychiatric Clinics of North America, 5, 715727.

Sheldon, S. H., Spire, J.-P. \& Levy, H. B. (1992) Sleep-wake schedule disorders. In Pediatric Sleep Medicine (eds S. H. Sheldon \& H. B. Levy), pp. 106-118. Philadelphia, PA: W. B. Saunders.

Simonds, J. F. \& Parraga, H. (1984) Sleep behaviors and disorders in children and adolescents evaluated at 
psychiatric clinics. Developmental and Behavioral Pediatrics, 5, 6-10.

Stores, G. (1991) Confusions concerning sleep disorders and the epilepsies in children and adolescents. British Journal of Psychiatry, 158, 1-7.

- (1992) Annotation: Sleep studies in children with a mental handicap. Journal of Child Psychology and Psychiatry, 33, 1303-1317.

- (1994) Investigation of sleep disorders including home monitoring. Archives of Disease in Childhood, 71, 184-185.

- (1996) Practitioner review: assessment and treatment of sleep disorders in children and adolescents. Journal of Child Psychology and Psychiatry, 37, 907-925.

— \& Crawford, C. (1998) Medical student education in sleep and its disorders. Journal of the Royal College of Physicians of London, 32, 149-153.

-, Ellis, A. J., Wiggs, L., et al (1998) Sleep and psychological disturbance in nocturnal asthma. Archives of Disease in Childhood, 78, 413-419.

- \& Wiggs, L. (1998) Abnormal sleep patterns associated with autism and related syndromes. Autism, 2, 157-169.

Stores, R. \& Wiggs, L. (1998) Sleep education in clinical psychology courses in the UK. Clinical Psychology Forum, 119, 14-18.

White, M. A., Williams, P. D., Alexander, D. J., et al (1990) Sleep onset latency and distress in hospitalised children. Nursing Research, 39, 134-139.

Wiggs, L. \& Stores, R. (1996) Sleep education in undergraduate psychology degree courses in the UK. Psychology Teaching Review, 5, 40-46.

- \& Stores, G. (1998) Behavioural treatment of sleep problems in children with severe learning disabilities and challenging daytime behaviour: effect on sleep patterns of mother and child. Journal of Sleep Research, 7, 119-126.

Wolfson, A., Lacks, P. \& Futterman, A. (1992) Effects of parent training on infant sleeping patterns, parents' stress and perceived parental control. Journal of Consulting and Clinical Psychology, 60, 41-48.

\section{Multiple choice questions}

1. Children with obstructive sleep apnoea:
a are not usually obese
b are mainly female
c often have enlarged tonsils and adenoids
d can be overactive rather than sleepy during the day
e often have Down's syndrome.

2. Sleeplessness problems in young children are often solved by:
a sedative medication
b setting limits
c introducing bedtime routines
d helping the child to fall asleep alone
e a large meal before going to bed.

3. Sleep requirements are increased in:
a delayed sleep phase syndrome
b rhythmic movements disorders
c narcolepsy
d adolescence
e Kleine-Levin syndrome.

4. Characteristic features of night terrors include:
a occurrence in the last third of the night
b amnesia for the events
c dream content
d family history
e abnormal EEG.

5. Sleep hygiene principles include:
a evening exercise
b strong chocolate drink at bed time
c unstimulating sleeping environment
d consistent bedtime
e avoiding late daytime naps.

$\begin{array}{llllllll}\text { MCQ answers } & & & & & \\ \text { 1 } & \text { 2 } & & 3 & & & & \\ \text { a T } & \text { a F } & \text { a F } & \text { a F } & \text { a F } \\ \text { b F } & \text { b T } & \text { b F } & \text { b T } & \text { b F } \\ \text { c T } & \text { c T } & \text { c T } & \text { c F } & \text { c T } \\ \text { d T } & \text { d T } & \text { d T } & \text { d T } & \text { d T } \\ \text { e F } & \text { e F } & \text { e T } & \text { e F } & \text { e T }\end{array}$

